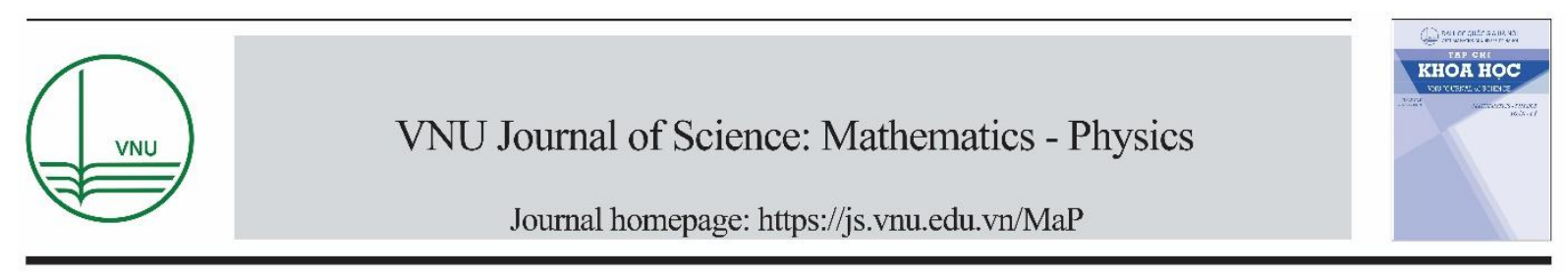

Original Article

\title{
Influences of PbS Quantum Dot Layers on Power Conversion Efficiency of Single Junction GaAs Solar Cells
}

\author{
Nguyen Dinh Lam* \\ Faculty of Engineering Physics and Nanotechnology, VNU University of Engineering and Technology, \\ 144 Xuan Thuy, Cau Giay, Hanoi, Vietnam
}

Received 10 July 2019

Revised 15 September 2019; Accepted 20 September 2019

\begin{abstract}
This paper investigates how PbS coating layers influence the characteristics of GaAs single junction solar cells through I-V characteristic measurements, optical reflectance spectra, and quantum efficiencies. To determine the expected influence, $\mathrm{PbS}$ quantum dots were coated on the surface of single junction GaAs solar cells by a drop coating method and the thickness of PbS quantum dot layer was controlled through changing the number of coating layers. The results show that, the short-circuit current can be improved up to $15 \%$ with two $\mathrm{PbS}$ coating layers. Other parameters such as $\mathrm{V}_{\text {oc }}$ and FF are hardly affected by the number of PbS coating layers. Based on the results of the optical reflectance spectra and quantum efficiencies, the enhancement in the shortcircuit current can be attributed to the antir-eflection of the $\mathrm{PbS}$ layers and the ability to transfer high energy photon-generated charge carriers.
\end{abstract}

Keywords: Quantum dots, solar cells, anti-reflection coating.

\section{Introduction}

When the light is illuminated on the surface of the solar cell, it is reflected by the Fresnel effect. The loss of light due to Fresnel reflections reduces the amount of photons absorbed, and thus reduces the power conversion efficiency of solar cell. Various methods have been proposed to limit the reflection of light at the surface of a solar cell as an antireflection coating (ARC) [1-4]. The basic principle of this method is to introduce an intermediate refractive index layer between the air and the material used to make the solar cell. Although ARC classes are now quite popular in the market, their performance in

\footnotetext{
*Corresponding author.

Email address: lamnd2005@gmail.com
}

https//doi.org/ 10.25073/2588-1124/vnumap.4361 
the ultraviolet (UV) band is still quite low. High-energy photons in this range are absorbed with a short absorption length so most of the high-energy photon generated carriers are trapped by defects on the solar cell surface, resulting in power conversion efficiency of the high-energy photons is negligible. Therefore, some studies have proposed using II-VI semiconductor nanoparticles or quantum dots (QDs) as a secondary source for the purpose of optimizing solar energy [5-8]. The use of QD materials has many advantages such as the ability to adjust the band gap of materials through fabrication process, high photon absorption performance, etc. The results also indicated that, the use of QDs as a secondary source has increased the ability of solar cells to convert energy.

\section{Experimental details}

\subsection{Single junction GaAs solar cells, PbS quantum dots}

The structure of a single junction GaAs solar cell is shown in Fig.1. The structure, from bottom to top, consists of n-type electrode (AuGe:Ni:Au), n-GaAs base, back surface field (BSF) n-AlGaAs, nGaAs base, $\mathrm{p}$-GaAs transmitter, $\mathrm{p}$-AlGaAs window, Ohmic contact $\mathrm{p}^{+}-\mathrm{GaAs}$, and $\mathrm{p}$-type electrode $(\mathrm{Ti} / \mathrm{Pt} / \mathrm{Au})$ layers. The fabrication processes of the devices were carried out in the following steps: (i) wet corrosion, (ii) n-type electrode evacuation, (iii) p-type electrode masking by photolithography, and (iv) p-type electrode deposition. The surface covering area of solar cells by electrodes accounts for 3.5\% of the total surface area of the solar cell (the surface area of the solar cell defined of $0.25 \mathrm{~cm}^{2}$ ). $\mathrm{PbS}$ quantum dots with a diameter of $6 \mathrm{~nm}$ used in this work were provided by Sigma-Aldrich.

\begin{tabular}{|c|c|c|}
\hline p-contact & Ti/Pt/Au (20/20/460) & $500 \mathrm{~nm}$ \\
\hline p-ohmic & $p+G a A s(Z n)$ & $300 \mathrm{~nm}$ \\
\hline Window & $\mathrm{p}-\ln G a P(Z n)$ & $50 \mathrm{~nm}$ \\
\hline Emitter & $\mathrm{p}-\mathrm{GaAs}(\mathrm{Zn})$ & $300 \mathrm{~nm}$ \\
\hline Base & $\mathrm{n}-\mathrm{GaAs}(\mathrm{Si})$ & $600 \mathrm{~nm}$ \\
\hline BSF & $n-\ln G a P(S i)$ & $50 \mathrm{~nm}$ \\
\hline Buffer & $\mathrm{n}-\mathrm{GaAs}(\mathrm{Si})$ & $200 \mathrm{~nm}$ \\
\hline Substrate & $n+\mathrm{GaAs} 2^{\circ}$ & 350 um \\
\hline n-contact & $\mathrm{AuGe} / \mathrm{Ni} / \mathrm{Au}(80 / 20 / 400)$ & $500 \mathrm{~nm}$ \\
\hline
\end{tabular}

Figure 1. Structure of single junction GaAs solar cell.

\subsection{Coating PbS quantum dot on the surface of single junction GaAs solar cells}

$\mathrm{PbS}$ QDs were directly coated on the surface of the single junction GaAs solar cell by drop-coating. The quantum dot solution concentration was controlled at $0.2 \mathrm{mg} / \mathrm{mL}$. The volume of solution for each coating is $20 \mu \mathrm{l}$.

\subsection{Investigation devices}

The I-V characteristic of solar cells was investigated under the illumination of the solar simulator using xenon lamps. The power of illuminated light was $100 \mathrm{~mW} / \mathrm{cm}^{2}$. Current and voltage values were 
collected using Keithley 2602A. The quantum efficiency is used to evaluate the generated carrier efficiency of the solar cell, which is determined by the ratio of the number of generated carriers on the number of incoming photons. Quantum efficiency was performed on the QX80 system. Optical reflectance spectrum was measured on UV-Vis system.

\section{Results and Discussion}

Fig. 2 (a) depicts I-V characteristic curves of a single junction GaAs solar cell depending on the number of $\mathrm{PbS}$ quantum dot coating layers. The results indicated that the open-circuit voltage $\left(\mathrm{V}_{\mathrm{oc}}\right)$ of the single junction GaAs solar cell is approximately $1 \mathrm{~V}$ and there is no significant change when the number of $\mathrm{PbS}$ coating layer changes. Therefore, the quantum dots layer on the surface of single junction GaAs solar cells does not affect on the open-circuit voltage or, in other words, the electrical and optical interactions of quantum dots do not influence on the structure of the p-n junction. The short-circuit current $\left(\mathrm{J}_{\mathrm{sc}}\right)$ has a marked change and was strongly dependent on the number of $\mathrm{PbS}$ quantum dot coating layers. The highest $\mathbf{J}_{\mathrm{sc}}$ value in this investigation is obtained when the number of $\mathrm{PbS}$ quantum dots is 2 layers. The change in $\mathrm{J}_{\mathrm{sc}}$ can be explained by the PbS quantum dot layer, which optimizes surface reflection as well as enhances the absorption of light in the ultraviolet region of solar radiation. This can be deeply explained by the result of external quantum efficiency (EQE) measurement.
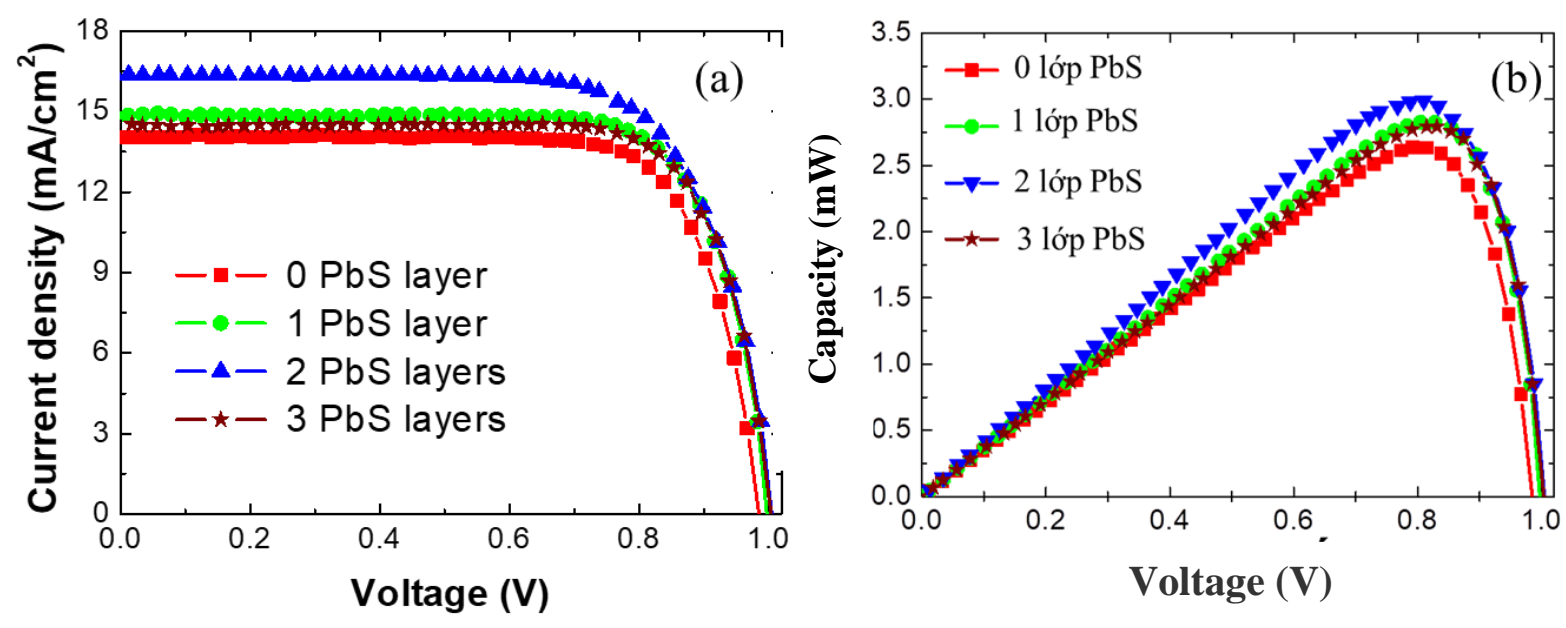

Figure 2. (a) J-V and (b) P-V characteristics of the cell with various in number of PbS QDs layers.

The maximum power $\left(\mathrm{P}_{\max }\right)$ of the cell was determined basing on the $\mathrm{P}-\mathrm{V}$ curves as shown in Fig.2(b). The obtained results indicated that the $\mathrm{P}_{\max }$ reached a maximum value of $3 \mathrm{~mW}$ in case two quantum dots layers was coated. Based on the working surface area of the cell, the maximum power density was found to be of $12 \mathrm{~mW} / \mathrm{cm}^{2}$. Furthermore, other characteristic parameters of the cells such as fill factor (FF) and power conversion efficiency $(\eta)$ can be also calculated according to the following formulas. The calculation result is shown in Table 1:

$$
\begin{aligned}
\mathrm{FF} & =\frac{P_{\max }}{U_{O C} I_{S C}}=\frac{I_{P \max } U_{P \max }}{U_{O C} I_{S C}} \\
\eta & =\frac{U_{O C} I_{S C} F F}{E_{i n} A}=\frac{U_{O C} J_{S C} F F}{E_{\text {in }}}
\end{aligned}
$$

Table 1. Characteristics of single junction GaAs solar cell depend on the number of $\mathrm{PbS}$ coating layers 


\begin{tabular}{lllll}
\hline Sample & $\mathrm{V}_{\mathrm{oc}}(\mathrm{V})$ & $\mathrm{J}_{\mathrm{sc}}\left(\mathrm{mA} / \mathrm{cm}^{2}\right)$ & $\mathrm{FF}(\%)$ & $\eta(\%)$ \\
\hline 0 PbS layer & 0.998 & 14.09 & 76.10 & 10.55 \\
1 PbS layer & 1.000 & 14.84 & 76.31 & 11.31 \\
2 PbS layers & 1.000 & 16.29 & 75.97 & 11.95 \\
3 PbS layers & 1.000 & 14.60 & 76.30 & 11.17 \\
\hline
\end{tabular}

The maximum power conversion efficiency of the cell is $11.95 \%$ corresponding to two $\mathrm{PbS}$ quantum dot coating layers. $\mathrm{V}_{\mathrm{oc}}=1.0 \mathrm{~V}, \mathrm{~J}_{\mathrm{sc}}=16.29 \mathrm{~mA} / \mathrm{cm}^{2}, \mathrm{FF}=75.97 \%$. The influence of the number of $\mathrm{PbS}$ quantum dot coating layers on the performance parameters of the cell can be more explained through the EQE spectrum.

Figure 3a shows the optical reflectance spectra of the cells depending on the number of quantum dot coating layers. The optical reflection at the surface of the cells is greatly reduced when it is coated with quantum dots and strongly depends on the number of $\mathrm{PbS}$ coating layer in the entire wavelength range from $300 \mathrm{~nm}$ to $900 \mathrm{~nm}$. The reducing optical reflection at the cell surface will cause the number of absorbed photons to increase, leading to more generated carriers, resulting in increased cell current density. The reducing optical reflection at the surface of the cell can be explained by reducing the refractive index difference between the surface of the cell and the environment by coating the quantum dot layer [8]. From the characteristic of EQE shown in Fig.3(b), when the PbS coating layer is thin (1 layer), the quantum efficiency of solar cells increases in the entire absorption range of the cell. This demonstrates that the quantum dot layer has played an important role in the transformation of ultraviolet region energy and the ability to optimize reflection at the surface, enhancing quantum efficiency in the entire region [9]. When the number of $\mathrm{PbS}$ quantum dots layer is 2 layers, the ability to transfer energy in the ultraviolet region tends to slightly decrease, but the quantum efficiency in the visible region is still improved. This makes the photo current density of this cell significantly increased. When the quantum dot layer is 3 layers, the quantum efficiency in the ultraviolet region decreases significantly. This can be explained when the quantum dot layer is thick enough, the ability to transfer the generated carriers by high energy photon absorption in the quantum dot layer decreases, the possibility of recombination the electron-hole pairs increases. This decline also entails a decrease in quantum efficiency in the visible region.
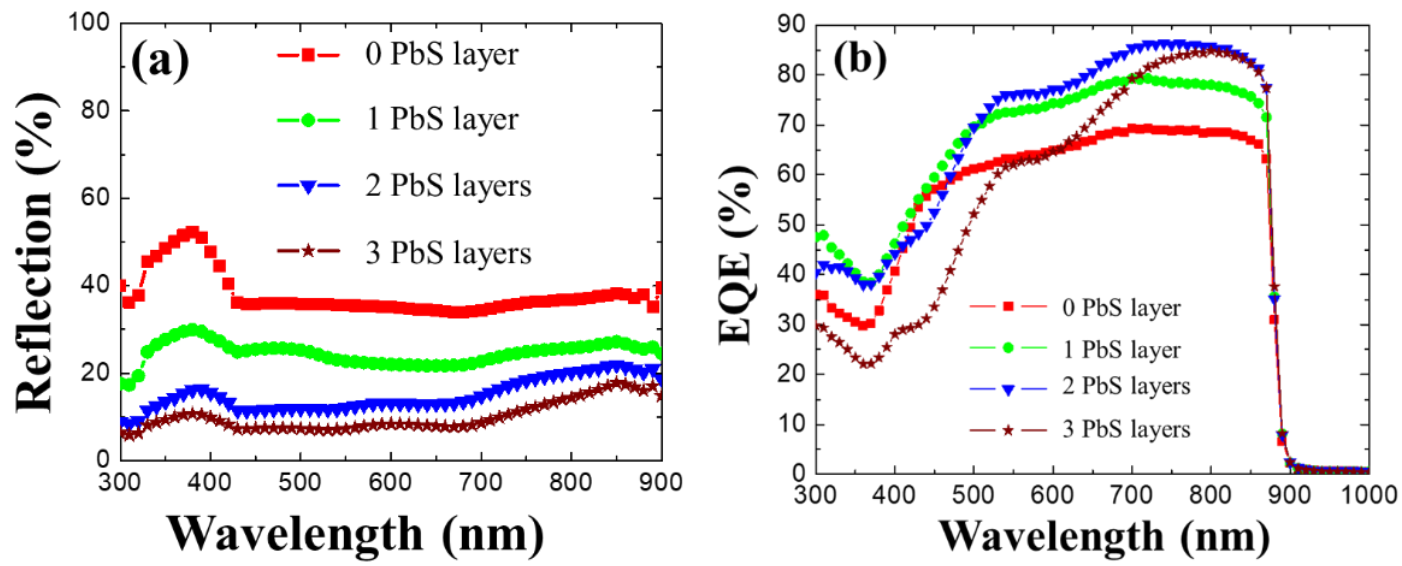

Figure 3. (a) Optical reflection and (b) EQE spectra of the cells depending on number of PbS layers. 
Figure 4 (a) shows the results of calculation the increase coefficient of quantum efficiency depending on the number of quantum dot coating layer. As mentioned, there are two main roles of quantum dot layer in increasing the power conversion efficiency of solar cells: (1) reducing optical reflectivity at the surface of the cell by reducing the degree of difference of refractive index; (2) enhance the generated carriers by optical absorption in ultraviolet region. The first role of quantum dot layer can be explained quite clearly through optical reflectance spectra. To clarify the second role of the quantum dot layer, we will determine the increase of internal quantum efficiency (quantum efficiency without reflection) through the following formula:

$$
\operatorname{IQE}(\lambda)=\frac{E Q E(\lambda)}{1-R(\lambda)}
$$

where, $R(\lambda)$ is the optical reflectance spectrum at the surface of the cell. Calculation results were redrawn and shown in Fig.4(b). When the quantum dot coating layer is 1 layer, the IQE enhancement coefficient in the ultraviolet region is larger than that of the cell without quantum dot coating layer. This demonstrates that the electronic pairs - holes produced by optical absorption in the quantum dot layer have been enhanced to increase the photocurrent density of the cell. As the number of quantum dots layer is increased, the thickness of quantum dot layer increases, leading to the moving distance of the photon generated carrier in the quantum dot layer increases. This makes it difficult to reach the electrodes of the cell. Most of them will be recombined and release solar energy causing energy loss. Therefore, as shown in Fig.4(b), when the quantum dot layer is 3 layers, there is no contribution of enhancement in the charge carriers by the quantum dots layer in ultraviolet region for the cell performance. Therefore, although the optical reflection at the surface of the cell is the lowest, however, the loss due to the moving distance increases, which results in a decrease in the power conversion efficiency of this cell.
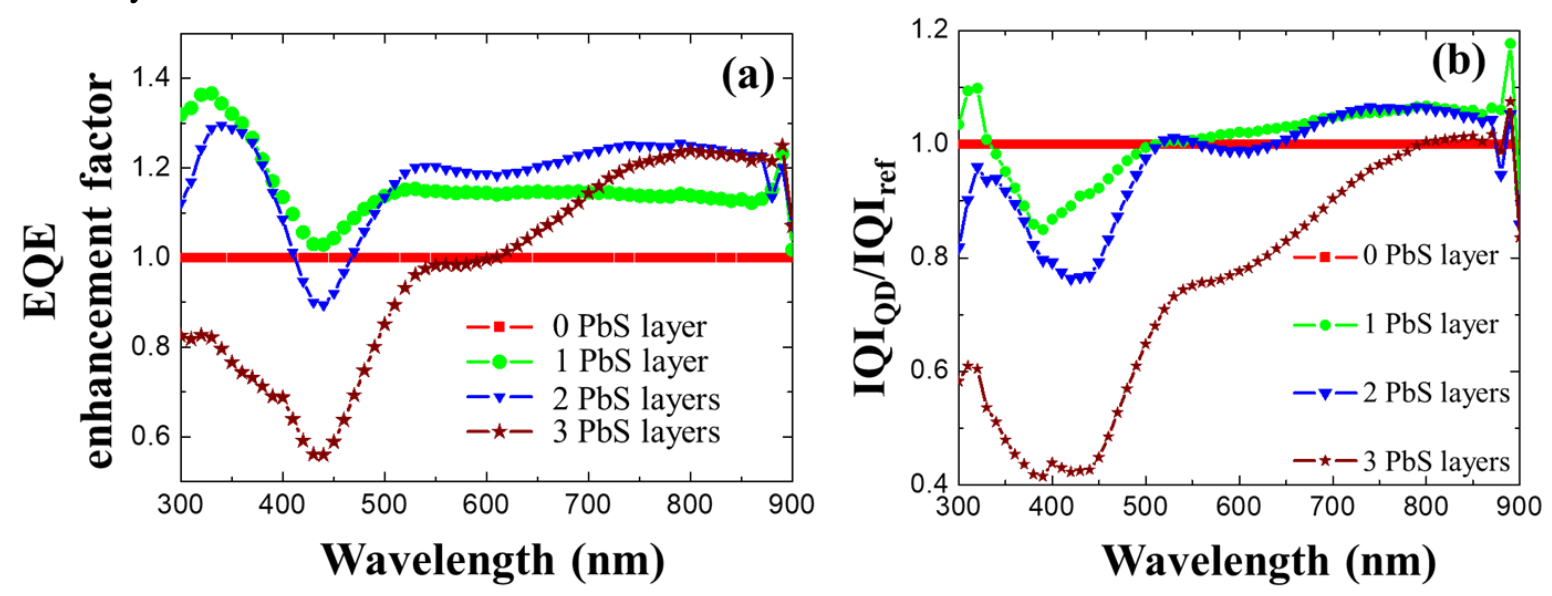

Figure 4. (a) EQE enhancement factor and (b) $\mathrm{IQI}_{\mathrm{QD}} / \mathrm{IQI}_{\mathrm{ref}}$ of the cell with the surface coated by $\mathrm{PbS}$ quantum dot layers

\section{Conclusions}

As obtained results, coating of $\mathrm{PbS}$ quantum dots can be utilized to improve performance parameters of solar cells. Herein, the improvement of the cell performance is mainly attributed to reducing the refractive index difference between the cell surface and environment, resulting in reducing the optical reflection at the surface of the cell. The enhancement of the generated carriers in the cell by the 
absorption of the ultraviolet radiation played an inconsiderable role. Therefore, it is necessary to carryout further studies for the enhancement in transferring carriers generated from the absorption of solar radiation by quantum dots layers.

\section{Acknowledgments}

This research is funded by Vietnam National University, Hanoi (VNU) under project number QG.19.20.

\section{References}

[1] K.W.A.Chee, Y. Hu, Design and optimization of ARC less InGaP/GaAs single-/multi-junction solar cells with tunnel junction and back surface field layers, Superlattices and Microstructures 119 (2018) 25-39. https://doi.org/10.1016/j.spmi.2018.03.071.

[2] Qiang-Ma, Wei-Jia Zhang, Deng-Hao Ma, Zhi-Qiang Fan, Xiao-Bo Ma, ZhaoyiJiang, Optimal design of quadruplelayer antireflection coating structure for conversion efficiency enhancement in crystalline silicon solar cells, Optik 177 (2019) 123-130. https://doi.org/10.1016/j.ijleo.2017.12.024.

[3] Avinash Kumar, Amartya Chowdhury, Reassessment of different antireflection coatings for crystalline silicon solar cell in view of their passive radiative cooling properties, Solar Energy 183 (2019) 410-418. https://doi.org/10.1016/j.solener.2019.03.060.

[4] N.Rezaei, O.Isabella, Z.Vroon, M.Zeman, Optical optimization of a multi-layer wideband anti-reflection coating using porous $\mathrm{MgF}_{2}$ for sub-micron-thick CIGS solar cells, Solar Energy 177 (2019) 59-67. https://doi.org/10.1016/j.solener.2018.11.015.

[5] S.M. Hubbard, C.D. Cress, C.G. Bailey, R. P. Raffaelle, S. G. Bailey, D. M. Wilt, Effect of strain compensation on quantum dot enhanced GaAs solar cells, Applied Physics Letters 92 (2008), 123512. https://doi.org/10.1063/1.2903699.

[6] Chien-Chung Lin, Hsin-Chu Chen, Yu Lin Tsai, Hau-Vei Han, Huai-Shiang Shih, Yi-An Chang, Hao-Chung Kuo, and Peichen Yu, Highly efficient CdS-quantum-dot-sensitized GaAs solar cells, Optics Express, 20(S2) (2012), A319. https://doi.org/10.1364/OE.20.00A319.

[7] Hau-Vei Han, Chien-Chung Lin, Yu-Lin Tsai, Hsin-Chu Chen, Kuo-Ju Chen, Yun-Ling Yeh, Wen-Yi Lin, Hao-Chung Kuo, Peichen Yu, A Highly Efficient Hybrid GaAs Solar Cell Based on Colloidal-Quantum-DotSensitization, Scientific Reports 4 (2014) 5734. https://doi.org/10.1038/srep05734.

[8] Hsin-Chu Chen, Chien-Chung Lin, Hau-Vei Han, Kuo-Ju Chen, Yu-Lin Tsai, Yi-An Chang, Min-Hsiung Shih, Hao-Chung Kuo, Peichen Yu, Enhancement of power conversion efficiency in GaAs solar cells with dual-layer quantum dots using flexible PDMS film, Solar Energy Materials and Solar Cells 104 (2012) 92-96. https://doi.org/10.1016/j.solmat.2012.05.003.

[9] Tzu-Neng Lin, Svette Reina Merden Santiago, Jie-An Zheng, Yu-Chiang Chao, Chi-Tsu Yuan, Ji-Lin Shen, Chih-Hung Wu, Cheng-An J.Lin, Wei-Ren Liu, Ming-Chiang Cheng, Wu-Ching Chou, Enhanced Conversion Efficiency of III-V Triple-junction Solar Cells with Graphene Quantum Dots, Scientific Reports 6 (2016) 39163. https://doi.org/10.1038/srep39163. 